\title{
Essais
}

ESSAIS

Revue interdisciplinaire d'Humanités

Hors-série 6 | 2021

Agrobiodiversité et territoires

\section{Réflexions autour de la création d'une filière de commercialisation de la venaison en Gironde}

Reflections on the creation of a marketing network for venison in Gironde

Carole Marin

\section{OpenEdition}

1 Journals

Édition électronique

URL : https://journals.openedition.org/essais/7496

DOI : $10.4000 /$ essais. 7496

ISSN : 2276-0970

Éditeur

École doctorale Montaigne Humanités

Édition imprimée

Date de publication : 1 mars 2021

Pagination : 89-110

ISBN : 978-2-492780-00-4

ISSN : $2417-4211$

Référence électronique

Carole Marin, "Réflexions autour de la création d'une filière de commercialisation de la venaison en Gironde », Essais [En ligne], Hors-série 6 | 2021, mis en ligne le 16 mars 2021, consulté le 18 janvier 2023. URL : http://journals.openedition.org/essais/7496 ; DOI : https://doi.org/10.4000/essais.7496 


\title{
Réflexions autour de la création d'une filière de commercialisa- tion de la venaison en Gironde
}

\author{
Carole Marin
}

En Europe, l'augmentation continue des tableaux de chasse au sanglier couplée à celle des impacts économiques, sanitaires, sécuritaires et écologiques liés à la présence de l'animal dans différents milieux suggère une croissance des populations de l'espèce au cours des dernières décennies ${ }^{1}$. La chasse représente le principal moyen de régulation des effectifs de sangliers ${ }^{2}$. Toutefois, la hausse des prélèvements de sangliers contraste avec la diminution du nombre de chasseurs : tandis qu'au sein de 16 pays européens analysés, dont la France, le nombre de sangliers prélevés par la chasse augmentait de $150 \%$ entre 1991 et 2011, les effectifs de chasseurs diminuaient en moyenne de $18 \%$. Dans le même temps, l'âge moyen des chasseurs augmentait dans la plupart des pays européens ${ }^{3}$.

Seulement $5 \%$ du volume de grand gibier chassé en France serait commercialisét, la venaison étant traditionnellement réservée aux chasseurs et à leurs proches. Mais les tableaux de chasse augmentant chaque année et les effectifs de chasseurs diminuant, il est probable que le monde de la chasse peine à " absorber " l'intégralité de la venaison dont il s'occupe seul de la préparation. En 2008, la Fédération Nationale des Chasseurs crée une marque déposée

1 Jürgen Tack, Wild Boar (Sus scrofa) populations in Europe: a scientific review of population trends and implications for management, Brussels, European Landowners' Organization, 2018, 56 p.

2 Oliver Keuling, Éric Baubet, Andreas Duscher, Cornelia Ebert, Claude Fischer, Andrea Monaco, Tomasz Podgórski, Céline Prevot, Katrin Ronnenberg, Gunter Sodeikat, Norman Stier, Henrik Thurfjell, "Mortality rates of wild boar Sus scrofa L. in central Europe ", European Journal of Wildlife Research, 59, 2013, p. 805-814.

3 Giovanna Massei, Jonas Kindberg, Alain Licoppe, Dragan Gacik, Nikica Šprem, Jiri Kamler, Éric Baubet, Ulf Hohmann, Andrea Monaco, Janis Ozolins, Sandra Cellina, Tomasz Podgorski, Carlos Fonseca, Nickolay Markov, Boštjan Pokorny, Carme Rosell, Andras Nahlik, « Wild boar populations up, numbers of hunters down? A review of trends and implications for Europe ", Pest Management Science, 71, 2015, p. 492-500.

4 Étienne Jobard, Jacques Marquay, Quentin Prigent, Stéphane Radureau, Marie-Laetitia des Robert, Evaluation du service écosystémique chasse en 2015, Rapport final d'étude Le Bipe, 20 novembre 2016, p. 26. 
"Gibier de chasse, chasseurs de France ", certifiant l'origine française du gibier vendu et mettant en avant les atouts nutritionnels et gustatifs de cette viande de gibier " naturelle ", destinée à un " consommateur qui veut un produit local et authentique. " ${ }^{5}$ Mais cette filière nationale serait insuffisamment organisée, et finalement 70 à $80 \%$ de la viande de gibier vendue en France serait importée 6

En parallèle, les dérives environnementales et sociales du modèle agroalimentaire conventionnel ainsi que les diverses crises sanitaires ont motivé une demande sociétale croissante pour des systèmes alternatifs ces dernières décennies ${ }^{7}$. En 2018, les achats de produits alimentaires issus de l'agriculture biologique en France représentaient un marché de plus de 9 milliards d'euros de chiffre d'affaires et affichaient une croissance de 15,4\% par rapport à l'année précédente ${ }^{8}$. Le développement de circuits courts de commercialisation $^{7}$, mais également de l'agriculture urbaine ${ }^{9}$, suggèrent aussi une aspiration de la société à reprendre le contrôle de son alimentation face à un modèle dominant productiviste ${ }^{10}$.

Forte de ces constats, la Fédération départementale des chasseurs des Pyrénées Orientales met en place en 2012 une filière locale de valorisation de la venaison ${ }^{11}$. Un marché de venaison d'origine polonaise était déjà installé dans le département. Un partenariat s'établit entre la Fédération départementale des chasseurs des Pyrénées Orientales, les abattoirs de Perpignan dans lesquels les contrôles sanitaires en vigueur sont effectués et l'entreprise locale de découpe et de transformation des viandes Guasch et Fils. À ce jour, le bilan est satisfaisant, les établissements Guasch et Fils ne rencontrant aucune difficulté à écouler la totalité de la venaison, essentiellement auprès de restaurateurs locaux, et dans une moindre proportion, auprès de bouchers de la région ${ }^{11}$. Une vidéo postée

5 Fédération Nationale des Chasseurs, "Le gibier de chasse : retrouvez les saveurs de la nature " [En ligne] Mis à jour le 09 mars 2020. Disponible sur : http://chasseurdefrance.com/le-gibierde-chasse-retrouvez-les-saveurs-de-la-nature/. Consulté le 11 mars 2020.

6 Fédération Nationale des Chasseurs, "Une consommation à développer " [En ligne] Mis à jour le 9 mars 2020. Disponible sur : http://chasseurdefrance.com/une-consommation-adevelopper/. Consulté le 11 mars 2020.

7 Élodie Brûlé-Gapihan, Audrey Laude, Étienne Maclouf, «Adopter une vision dynamique de l'innovation soutenable : le cas des circuits courts alimentaires ", Revue d'économie industrielle, 159, 2017, p. 53-79.

8 AND International, Observatoire National de l'Agriculture Biologique, Le marché alimentaire bio en 2018, estimation de la consommation des ménages en produits alimentaires biologiques en 2018, Rapport d'octobre 2019, p. 5, 65 p. Disponible sur : https:/www.agencebio.org/vosoutils/les-chiffres-cles/. Consulté le 30 avril 2020.

9 Paula Nahmias, Yvon Le Caro, "Pour une définition de l'agriculture urbaine : réciprocité fonctionnelle et diversité des formes spatiales ", Environnement urbain, 6, 2012, p. 1-16.

10 Nicolas Bricas, Claire Lamine, François Casabianca, «Agricultures et alimentations : des relations à repenser? " Natures Sciences Sociétés, 21, 2013, p. 66-70.

11 Fédération Départementale des Chasseurs des Pyrénées-Orientales, Schéma Départemental de Gestion Cynégétique des Pyrénées-Orientales 2016-2022, 2016, p. 151-152. 
par la Fédération des chasseurs des Pyrénées Orientales montre qu'au-delà de retombées économiques de cette filière pour les différents acteurs, ces derniers éprouvent une fierté à travailler un produit qu'ils considèrent comme de qualité et authentique. Stéphane Guasch, gérant des établissements éponymes, insiste sur l'importance de proposer un produit issu d'un circuit très court, un moyen selon lui de "se battre pour le département». Deux restaurateurs quant à eux mettent en avant l'origine locale de la viande, gage de fraîcheur, de qualité et de traçabilité " on sait ce qu'on propose à nos clients ", énoncent les qualités nutritionnelles du gibier prélevé à l'état sauvage " dans la mesure où c'est sauvage, c'est bio, il se nourrit lui-même ", "c'est une viande saine, non grasse ", et finalement expriment leur satisfaction de proposer un produit "chassé par nos chasseurs, comme le faisaient nos arrières grands-parents $»^{12}$.

L'article propose une discussion autour de la mise en place d'une filière de commercialisation de la venaison de sanglier dans le contexte girondin. Nous examinons plus particulièrement les possibilités d'intégration d'un tel projet à celui de la gestion cynégétique des populations de sangliers présentes dans l'enveloppe urbaine bordelaise, initié en 2019 par la Fédération des chasseurs de la Gironde. La réflexion est centrée sur la confrontation des différents points de vue des acteurs du monde de la chasse girondine quant à l'émergence de pratiques cynégétiques urbaines et la mise en place d'une filière de distribution de la venaison.

L'analyse des discours tenus par les responsables cynégétiques lors de l'Assemblée Générale de la Fédération départementale des chasseurs de la Gironde du 27 avril 2019 occupe une place centrale dans cet article. Cet événement annuel rassemble personnalités politiques (préfet, sénateurs, députés), gestionnaires, administrateurs cynégétiques et chasseurs girondins. Les tours de parole se succèdent, ils portent, suivant le locuteur, sur des considérations politiques, techniques, sociales ou encore domestiques relatives à la question cynégétique et à celle de la gestion de la faune. Cette rencontre offre au chercheur une occasion unique de saisir les enjeux politiques et sociaux inhérents au modèle cynégétique local. L'analyse d'extraits de discours relevés lors de l'assemblée, complétés par ceux obtenus lors d'entretiens individuels réalisés auprès des gestionnaires de la Fédération départementale des chasseurs de la Gironde dans le cadre de la recherche, s'articule aux résultats plus quantitatifs d'une enquête adressée aux chasseurs girondins. En effet, si l'échantillon de personnes présentes lors de l'assemblée générale des chasseurs de la Gironde du 27 avril 2019 reflète la diversité des acteurs du monde cynégétique girondin, les points de vue recueillis sont très majoritairement ceux exprimés par les gestionnaires cynégétiques de la Fédération des chasseurs girondins. Pourtant,

12 Fédération Départementale des Chasseurs des Pyrénées-Orientales, "La filière de valorisation de la venaison » [Vidéo en ligne] 12 avril 2016. Disponible sur : https://www.youtube.com/ watch?v=vNT0g9U2Ems. Consultée le 12 mars 2020. 
il est possible que les opinions et objectifs des gestionnaires ne soient pas partagés par la population de chasseurs girondins. Un formulaire, numérique, est donc envoyé le 24 janvier 2020 aux 21440 chasseurs ayant validé leur permis de chasser auprès de la Fédération départementale des chasseurs de la Gironde pour la saison cynégétique 2019-2020 et pour lesquels nous disposons d'une adresse mail valide (soit 58,3\% des 36263 chasseurs girondins). Deux mois après la diffusion de l'enquête, 4581 observations sont récoltées. Après suppression des doublons, des observations correspondant aux chasseurs ne résidant pas en Gironde ainsi que de celles pour lesquels l'âge, le genre et la commune de résidence ne sont pas renseignés, et après redressement de l'échantillon à partir de l'arrondissement de résidence et d'un quota croisé entre le genre et les classes d'âges, 2869 observations sont retenues.

La réflexion autour de la commercialisation de la venaison nous a été inspirée par notre double formation : en médecine vétérinaire et, plus récemment, en géographie. Ainsi, le premier temps de l'article expose de façon pragmatique les moyens nécessaires à la création d'une filière locale urbaine de commercialisation de la venaison de sanglier à Bordeaux et dégage les atouts potentiels théoriques de la filière, perçus comme cohérents avec les objectifs annoncés par la Fédération des chasseurs de la Gironde. Dans le second temps de l'article, l'impact de l'introduction de cette filière urbaine sur le fonctionnement sociétal est discuté : la perspective rationalisante de l'expertise rencontre-t-elle celle des responsables cynégétiques fédéraux, des chasseurs, et de la société urbaine?

\section{Le contexte girondin}

\section{La gestion des populations girondines de sanglier}

En Gironde, la saison de chasse au sanglier s'étend du 15 août au 28 février de l'année suivante. Du $1^{\text {er }}$ juin au 14 août, la chasse au sanglier peut être pratiquée en battue, à l'affût ou à l'approche sur autorisation préfectorale individuelle délivrée au détenteur du droit de chasse après avis du Président de la Fédération départementale des chasseurs. On parle alors de tirs d'été de régulation. Du $1^{\text {er }}$ au 31 mars, des battues administratives au sanglier sont organisées par le préfet à des fins de destruction, l'animal passant alors d'un statut de " gibier " à celui d' " espèce susceptible d'occasionner des dégâts " ${ }^{13}$. Au cours des mois d'avril et de mai, ce sont les lieutenants de louveterie, auxiliaires de l'État nommés par le préfet, qui sont en charge de la régulation administrative des populations de sangliers. Dans les zones non chassées telles que l'enveloppe urbaine bordelaise, ces derniers interviennent tout au long

13 Préfet de la Gironde, Campagne de chasse 2018-2019. Arrêté relatif aux dates d'ouverture et de clôture de la chasse pour la campagne 2018-2019 dans le département de la Gironde, 2018, 6 p. 
de l'année pour réguler les populations de sanglier. Ces mesures, soumises à arrêté préfectoral, sont de deux types : soit les individus sont appâtés, piégés puis abattus, soit ils sont " détruits " lors de battues administratives.

Les prélèvements de sangliers réalisés sur le département n'ont cessé d'augmenter au cours des dernières années. Pour la saison cynégétique 20182019, les tableaux de chasse au sanglier comptabilisent 10978 prélèvements du $1^{\text {er }}$ juin 2018 au 28 février 2019, auxquels s'ajoutent 1251 prélèvements administratifs réalisés au cours du mois de mars 2019 et 665 sangliers tués par les lieutenants de louvèterie sur la période s'étendant du $1^{\text {er }}$ juillet 2018 au 30 juin 2019. Cinq ans plus tôt, la saison cynégétique 2013-2014 totalisait 7761 prélèvements de sangliers, toutes catégories confondues ${ }^{14}$, les prélèvements ont augmenté de $66 \%$ sur la période. De plus, la confrontation de la courbe des effectifs de chasseurs girondins avec celle des prélèvements de sangliers traduit une situation qui semble échapper au dispositif de régulation du Plan National de Maîtrise du Sanglier instauré en 2009 ${ }^{15}$. Tandis que la population des chasseurs a été divisée par plus de deux en 40 ans (Figure 1), la courbe des prélèvements de sangliers suit une orientation inverse : ces 20 dernières années, les prélèvements de sangliers réalisés par les chasseurs ont été multiplié plus de 8 fois (Figure 2). Enfin, alors que la tranche d'âge la plus représentée dans la population de chasseurs girondins en 2010 était celle de 51 à 60 ans, elle correspond, dix ans plus tard, à celle de 61 à 70 ans (Figure 3), suggérant un faible renouvellement des effectifs de chasseurs ${ }^{16}$.

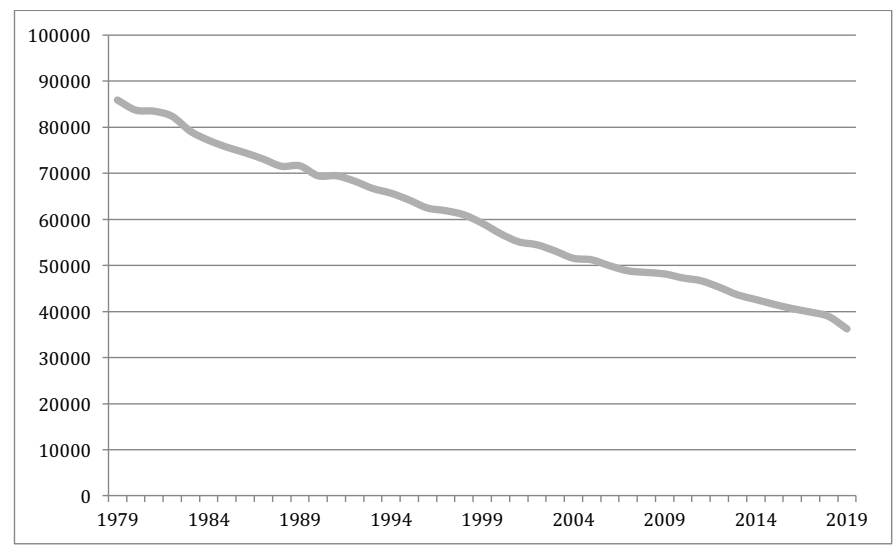

Figure 1 : 40 ans d'évolution des effectifs de chasseurs en Gironde (d'après les données récoltées auprès de la Fédération départementale des chasseurs de la Gironde, 2019)

14 D'après les données récoltées auprès de la Fédération départementale des chasseurs de la Gironde, 2019.

15 Ministère de l'Écologie, de l'Énergie, du Développement Durable et de la Mer, en charge des Technologies vertes et des Négociations sur le climat, Plan National de Maîtrise du Sanglier. Un cadre d'actions techniques pour agir au plan départemental, 2009, 25 p.

16 Fédération départementale des chasseurs de la Gironde, 2019, loc. cit. 


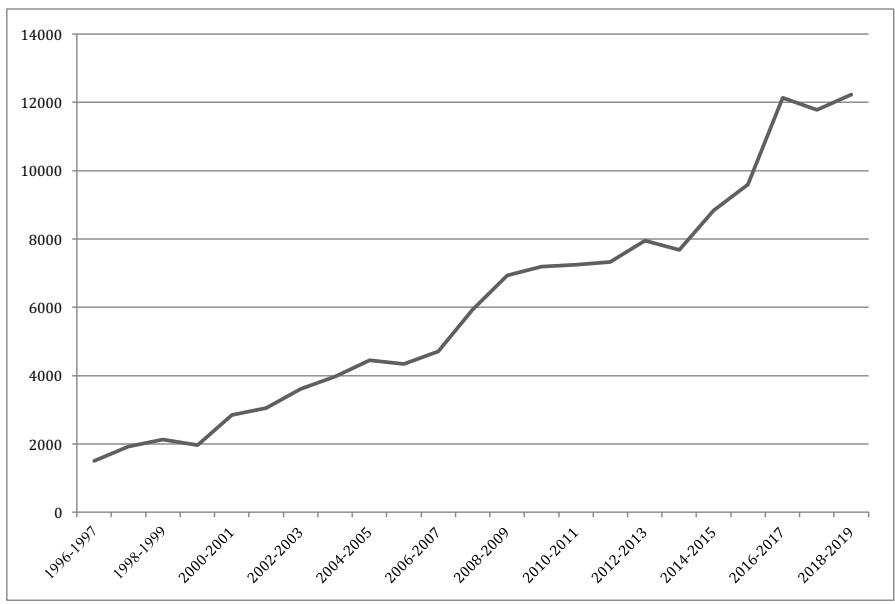

Figure 2 : Les prélèvements de sangliers depuis 20 ans en Gironde ( $d$ 'après les données récoltées auprès de la Fédération départementale des chasseurs de la Gironde, 2019)

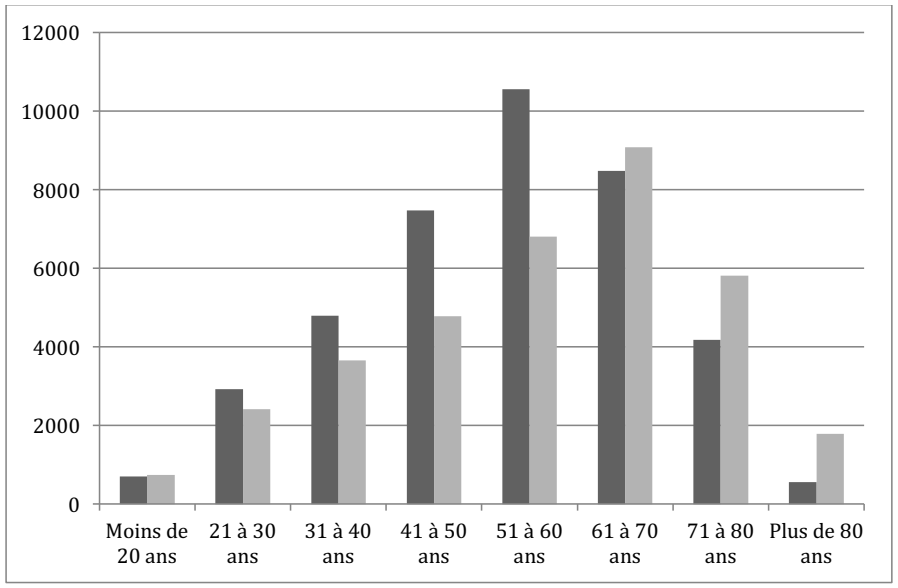

Figure 3 : Répartition des adhérents à la Fédération des chasseurs de Gironde par tranche d'âge en 2010 (gris foncé) et en 2020 (gris clair)

(d'après les données récoltées auprès de la Fédération départementale des chasseurs de la Gironde, 2020)

\section{Les circuits de la venaison}

À l'issue de la chasse, les chasseurs se partagent la venaison, tandis que les carcasses de sangliers prélevés à des fins de destruction sont destinées à être incinérées, la venaison n'étant ni consommée ni valorisée. Selon la réglementation en vigueur, les chasseurs sont autorisés à livrer leur gibier en vue de la consommation humaine selon différents circuits. Le plus souvent, la venaison est réservée à l'autoconsommation. Dans ce cas, elle est préparée et consommée 
par le chasseur, seul, en famille ou en compagnie des chasseurs ayant participé à la chasse, et aucune réglementation particulière ne s'applique. S'il offre sa venaison à un proche, le chasseur a l'obligation d'informer le consommateur des risques liés à la trichinellose, maladie parasitaire transmissible à l'homme par ingestion de viande infestée ${ }^{17}$. La fédération nationale des chasseurs a fait réaliser à cet effet des sacs à gibier portant la mention « Le sanglier peut être porteur d'un parasite : la trichine. C'est pourquoi la viande de sanglier doit toujours être bien cuite à cœur ! » La venaison peut être servie dans le cadre d'un repas de chasse, ouvert au public, ou dans le cadre d'un repas associatif, pour lequel le chasseur livre des carcasses éviscérées entières, l'ensemble de la préparation (dépouille, découpe, congélation, cuisson) revenant à l'organisateur du repas. Le chasseur a également la possibilité de vendre sa venaison à un commerce de détail local, fournissant directement le consommateur final. Ce commerce doit se trouver dans un rayon de $80 \mathrm{~km}$ du lieu de chasse. Pour ces trois derniers cas, l'examen initial de la venaison par une personne ayant suivi la formation "hygiène de la venaison " et la recherche de trichine par un laboratoire agréé sont obligatoires. Enfin, un circuit long de commercialisation de la venaison est possible, par la vente de la carcasse à un atelier de traitement du gibier agréé, éventuellement par le biais d'un collecteur. Dans tous les cas, la traçabilité de la venaison est obligatoirement assurée de l'action de chasse au dernier détenteur avant le consommateur final.

En Gironde, il n'existe pas de filière organisée de commercialisation de la venaison. Le partage et la dégustation du gibier faisant partie de l'activité de chasse, il n'est pas envisageable de commercialiser la totalité de la venaison prélevée par les chasseurs sur le département. Néanmoins, la tendance à l'augmentation des prélèvements de sangliers couplée à la diminution des effectifs de chasseurs girondins justifie l'étude des moyens nécessaires à la mise en place d'une filière facilitant le traitement et la valorisation de la venaison, susceptible d'intéresser certaines associations de chasse qui ont atteint les limites de la consommation familiale et d'investissement humain pour préparer la venaison.

\section{Les moyens nécessaires à la création d'une filière de valorisation de la venaison}

Le paragraphe qui suit propose de retracer le circuit d'un sanglier prélevé intégré dans une filière hypothétique de traitement et valorisation de la venaison en Gironde respectant les dispositions réglementaires de l'arrêté du 18 décembre 2009 relatives aux viandes fraîches de gibier sauvage ${ }^{18}$.

17 Jean Hars, Sophie Rossi, "Évaluation des risques sanitaires liés à l'augmentation des effectifs de sangliers en France ", Faune sauvage, 288, 2010, p. 23-28. 
L'animal chassé dans les territoires de chasse des associations adhérentes au projet est impérativement marqué, sur le site de prélèvement, d'un bracelet d'identification délivré par la Fédération départementale des chasseurs de la Gironde. L'animal entier est ensuite rapidement transporté vers un centre de collecte, pouvant être commun à plusieurs organisations cynégétiques de la zone. L'éviscération et l'examen initial de la venaison et des abats par une personne formée sont alors réalisés dans l'espace adapté et prévu à cet effet au centre de collecte. En cas d'anomalie constatée lors de l'examen initial, les abats rouges doivent accompagner la carcasse et être précisément identifiés pour subir une inspection post-mortem officielle au centre de traitement. Les numéros d'identifications des bracelets de marquage sont repris sur les fiches d'examen initial ou fiches accompagnant obligatoirement la carcasse tout au long du circuit. Cette mesure, de même que la tenue de registres internes au centre de collecte, garantissent la traçabilité de la carcasse et s'inscrivent dans la lutte contre le braconnage. La société d'équarrissage assure la prise en charge des déchets de venaison, tandis que le transporteur achemine la carcasse éviscérée et non dépouillée en camion réfrigéré vers le centre de traitement, qui se charge alors du dépeçage de l'animal et des contrôles sanitaires en vigueur. Pour le sanglier, l'examen post-mortem après dépeçage et la recherche de trichine sont obligatoires. Toutes les pièces de grand gibier destinées à la consommation humaine entrées en atelier de traitement doivent être revêtues d'une marque de salubrité européenne sous la responsabilité du vétérinaire officiel du centre. Après maturation de la viande en chambre froide pendant au minimum une semaine, la découpe, le parage, le désossage et le conditionnement de pièces bouchères ou la préparation de produits charcutiers s'effectuent au sein d'un atelier de découpe et de transformation de la viande. Finalement, les détaillants (restaurateurs, bouchers, charcutiers) achètent la venaison préparée pour la proposer au consommateur final.

Pour la création d'une filière locale et contrôlée de commercialisation de la venaison, un partenariat entre la Fédération des chasseurs de la Gironde coordinatrice du projet, une société d'équarrissage, une société de transport et un centre de traitement du gibier devrait donc être envisagé. Les organisations de chasse du département choisissant d'adhérer au programme s'engageraient alors à fournir un certain nombre de carcasses par saison cynégétique, en respectant les dispositions prévues par le cahier des charges de la filière. Cette quantité serait déterminée par les associations de chasse elles-mêmes et réévaluée chaque année en fonction de l'évolution des prélèvements de sangliers et de la consommation du gibier par ses adhérents. 


\section{Du projet de gestion à la création d'une filière}

\section{Les projets prioritaires}

À l'heure actuelle, les préoccupations des gestionnaires de la Fédération des chasseurs de la Gironde se tournent vers deux phénomènes, faisant l'objet de deux projets de gestion distincts.

Une première priorité est la maîtrise du risque sanitaire pour les espèces domestiques voire pour l'Homme accompagnant la prolifération de l'espèce ${ }^{19}$. En septembre 2018, la Belgique déclare cinq cas de peste porcine africaine (PPA) chez des sangliers, à proximité de la frontière avec la France. Immédiatement, la France met en place une zone de lutte renforcée dans les départements frontaliers, sur le "front " de la $\mathrm{PPA}^{20}$. La maladie n'est pas une zoonose. Néanmoins, son arrivée sur le territoire français entraînerait de lourdes conséquences économiques liées à un fort taux de mortalité dans les élevages porcins. D'autant plus que le statut indemne de peste porcine de la France lui permet d'exporter $40 \%$ de sa production porcine ${ }^{21}$. D'autre part, la Nouvelle-Aquitaine représente la région la plus touchée actuellement par la tuberculose bovine, comptant $91 \%$ des foyers détectés sur le territoire nationa ${ }^{22}$. Le sanglier, le blaireau et le cerf peuvent constituer un réservoir de la maladie ${ }^{23}$. "Avec l'arrivée de la peste porcine africaine aux portes du territoire national et le classement de quarante communes de Gironde en zone à risque de tuberculose bovine, nous devons passer d'une gestion des déchets que l'on pouvait qualifier d'un peu artisanale jusqu'à présent à un partenariat avec la Direction Départementale de Protection des Populations et des sociétés d'équarrissage pour une collecte organisée des déchets de venaison. ${ }^{24}$ Jusqu'alors, les déchets de venaison, représentant 350 tonnes par an pour le département de la Gironde, étaient enfouis. Face à ces nouveaux enjeux sanitaires, la Fédération des chasseurs de la Gironde met en place trois

19 Jean Hars et Sophie Rossi, Faune sauvage, 2010, loc. cit.

20 Anne Bronner, Didier Calavas, Julien Cauchard, Pascal Hendrikx, Alizé Mercier, MarieFrédérique Le Potier, Peste porcine africaine : apparition en Belgique, progression en Europe de l'est et en Chine. Note d'information du 19 septembre 2018, Veille Sanitaire Internationale, Plateforme ESA, 3 p.

21 Ministère de l'Agriculture et de l'Alimentation. Plan d'actions. Organisation de la prévention, de la surveillance et de la lutte contre la peste porcine africaine. 2018, $12 \mathrm{p}$.

22 Fanny Pandolfi, Lisa Cavalerie, Fabrice Chevalier, Pierre Jabert, Didier Calavas, JeanJacques Bénet, Nicolas Keck, Sébastien Girard, Anne-Laure Lefebvre, Franck Martin, Édouard Reveillaud, Stéphanie Philizot, Barbara Dufour, Anne Bronner, Surveillance de la tuberculose bovine en 2018 (point au 4 avril). Note d'information du 24 avril 2018, Veille Sanitaire Internationale, Plateforme ESA, 10 p.

23 Barbara Dufour, Jean-Jacques Bénet, "L'infection à Mycobacterium bovis en France en 2014 : recrudescence et inquiétudes ", Revue Francophone des Laboratoires, 472, 2015, p. 67-75.

24 Michel Massias, administrateur responsable de la Commission Équarrissage, Assemblée générale de la Fédération départementale des chasseurs de la Gironde du 27 avril 2019. 
obligations dans la zone à risque de tuberculose : l'inspection systématique des carcasses par une personne ayant suivi la formation "hygiène de la venaison " dispensée gratuitement par la Fédération des chasseurs de la Gironde, la déclaration de toute lésion suspecte à la Direction Départementale de Protection des Populations et le dépôt des déchets de venaison dans les points de collecte mis à disposition par les services d'équarrissage. Il est prévu que ces dispositions s'étendent à tout le département au cours de l'année $2020^{25}$.

Le contrôle de la pénétration de populations de sangliers au sein d'espaces urbains et péri-urbains représente une deuxième priorité pour les gestionnaires. Ces territoires se retrouvent aujourd'hui confrontés à des enjeux économiques (dégâts dans les exploitations agricoles urbaines et périurbaines, dans les espaces verts aménagés, dans les jardins de particuliers), écologiques (envahissement de petites réserves urbaines), de sécurité (collisions automobiles, rencontres avec des humains ou des animaux domestiques) et sanitaires, liés à la présence de cet animal sauvage ${ }^{26}$. Ces enjeux étaient autrefois réservés aux seuls espaces ruraux. «Dans les territoires péri-urbains, le temps des inventaires et des audits est derrière nous! Il est question d'unités de gestion et de plans de gestion des populations. ${ }^{27}$ À Bordeaux, un projet de gestion cynégétique a vu le jour en 2019. L'objectif est la mise en place de l'association de chasse péri-urbaine de Bordeaux (ACPB), pilotée par la Fédération départementale des chasseurs de la Gironde, au sein d'une nouvelle unité de gestion " périurbaine " concernant 31 communes de l'enveloppe urbaine bordelaise dans lesquelles 6500 hectares chassables ont été identifiés (Carte 1) ${ }^{28}$. Au-delà de la maîtrise des enjeux liés à la présence de sangliers au sein du territoire concerné par le projet, les gestionnaires perçoivent une opportunité de recrutement de nouveaux chasseurs à travers l'ouverture de territoires de chasse en zone urbaine : «La métropole bordelaise prend 10000 habitants par an, peut-être y a-t-il des chasseurs parmi eux. $»^{29}$ Les modes de chasse aux sangliers traditionnels, collectifs, bruyants et nécessitant de grands espaces tels que la battue

25 Ibid.

26 Carles Conejero, Raquel Castillo-Contreras, Carlos González-Crespo, Emmanuel Serrano, Gregorio Mentaberre, Santiago Lavín, Jorge Ramón López-Olvera, "Past experiences drive citizen perception of wild boar in urban areas ", Mammalian Biology, 96, 2019, p. 68-72 ; Stéphane Builles, gestionnaire de la réserve naturelle des marais de Bruges, entretien du 3 décembre 2019 ; Xavier Fernandez-Aguilar, Marcelo Gottschalk, Virginia Aragon, Jordi Camara, Carmen Ardanuy, Roser Velarde, Nuria Galofre-Mila, Raquel Castillo-Contreras, Jorge R. Lopez-Olvera, Gregorio Mentaberre, Andreu Colom-Cadena, Santiago Lavin, Oscar Cabezon, «Urban Wild Boars and Risk of Zoonotic Streptococcus suis, Spain », Emerging Infectious Disease, 24(6), 2018, p. 1083-1086.

27 Jérôme Martin, administrateur responsable de la Commission Péri-urbain, Assemblée générale de la Fédération départementale des chasseurs de la Gironde du 27 avril 2019.

28 D'après les discussions avec Emmanuel Robin, directeur des actions territoriales de la Fédération départementale des chasseurs de la Gironde et en charge du projet de création de l'ACPB, 2019.

29 Jérôme Martin, Assemblée générale de la Fédération départementale des chasseurs de la Gironde, 27 avril 2019, loc. cit. 


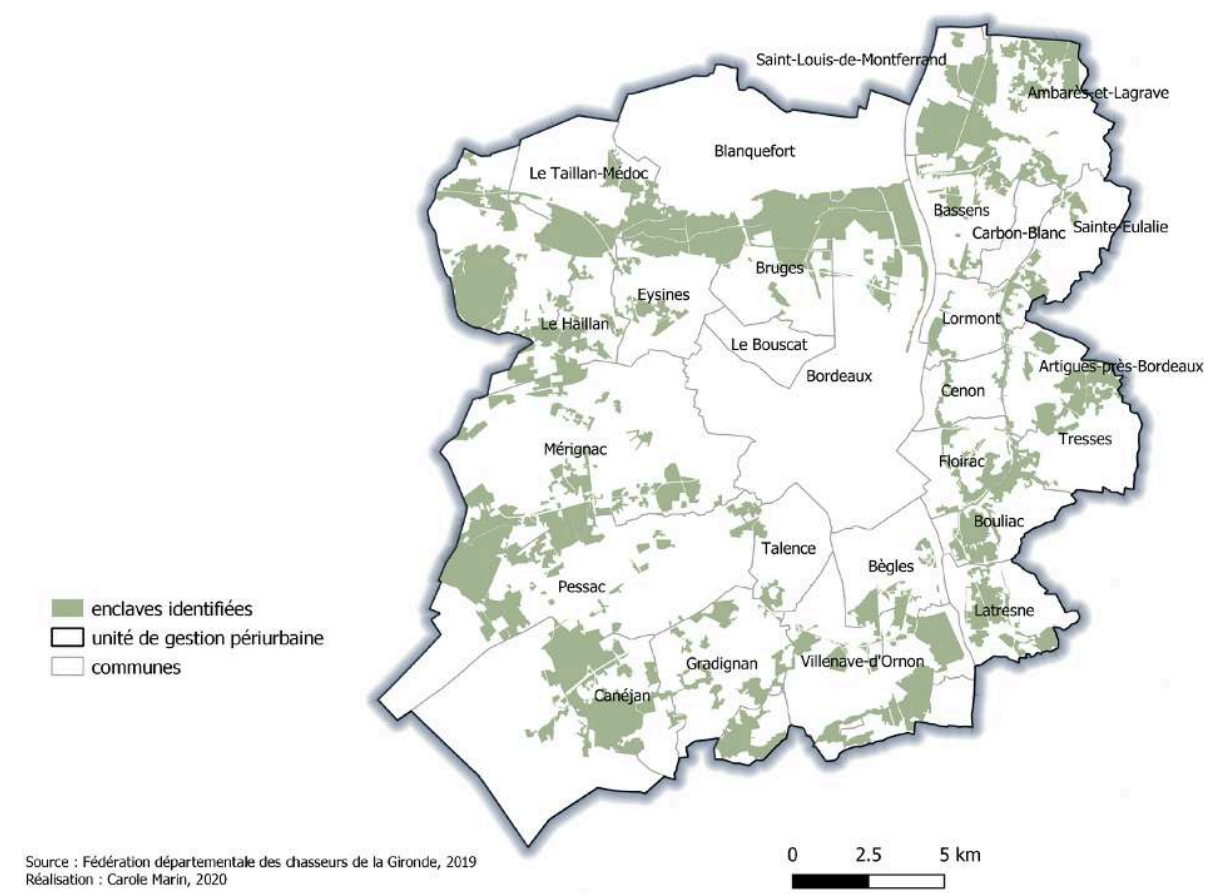

Carte 1 : Enclaves, définies comme des espaces potentiellement chassables et susceptibles d'abriter des populations d'espèces de grand gibier, de l'enveloppe urbaine de Bordeaux (d'après les données récoltées auprès de la Fédération départementale des chasseurs de la Gironde, 2019)

aux chiens courants, laissent place dans ces zones urbanisées à des pratiques individuelles telles que la chasse à l'approche ou à l'affut, à la carabine ou à l'arc ${ }^{30}$. En permettant une certaine souplesse dans la pratique, la chasse à l'approche ou à l'affût seraient plus compatibles avec les modes de vie actuels : "Le monde change. Il y a 50 ans, la pratique de la battue toute la journée du samedi et du dimanche posait moins de problèmes qu'aujourd'hui. De nos jours, ces journées correspondent aux moments que l'on peut passer en famille, donc forcément si on les passe en battues, des tensions peuvent facilement apparaître dans le couple. En revanche, l'affût, l'approche, sont tout à fait adaptés à ces nouveaux fonctionnements familiaux puisque ce sont des pratiques qui permettent d'y aller 2 heures le samedi, puis on est libre soit le matin, soit l'après-midi pour passer du temps en famille. On peut y retourner 2 ou 3 heures le dimanche. $»^{31} \AA \grave{A}$ travers l'insertion de ces pratiques autrefois marginales dans le paysage cynégétique girondin, les gestionnaires espèrent donc attirer une nouvelle catégorie de chasseurs au sanglier. Enfin, concernée par l'image de la chasse dans la société « Nous sommes à l'époque du chasse

30 D'après les discussions avec Emmanuel Robin, 2019, loc. cit.

31 Henri Sabarot, président de la Fédération départementale des chasseurs de la Gironde, Remise des diplômes aux lauréats du brevet grand gibier de l'Association départementale des chasseurs de grand gibier de Gironde, 17 novembre 2019. 
bashing, où l'on donne de plus en plus de tribunes aux anti-spécistes et aux vegans, mais cela ne nous affaiblira pas ${ }^{32}$, la Fédération considère la pratique de la chasse à l'arc comme porteuse d'une " très bonne image " ${ }^{33}$, susceptible d'être mieux acceptée par les habitants de ces zones particulières : "dans les milieux urbains et périurbains, on a à faire à des citadins qui ne comprennent pas la carabine, les chiens. On prône l'approche et l'affût pour ces milieux et en particulier la chasse à l'arc qu'on essaye de développer, d'accompagner et qui est totalement adaptée à ces milieux. $»^{34}$

Dans l'enveloppe urbaine bordelaise, la gestion des populations de sangliers est administrative. Ce processus déshumanisé mais néanmoins peu visible, sans publicité et donc non sujet à polémique, pourrait laisser place à la pratique cynégétique dans le paysage urbain. Le discours de Fabienne Buccio, alors récemment nommée préfète de la Gironde, lors de l'assemblée générale des chasseurs de la Gironde du 27 avril 2019, illustre l'inquiétude du pouvoir central face au phénomène de pénétration de populations de sangliers au sein de zones fortement urbanisées. Elle annonce un « soutien total pour la gestion coordonnée dans le périurbain " par la nouvelle organisation cynégétique périurbaine, à laquelle elle attribue un double rôle : celui de la régulation des populations de sangliers et celui d'une offre de chasse nouvelle, qu'elle considère alors comme social. Finalement, l'ouverture de nouveaux territoires de chasse en milieux urbains et péri-urbains associée aux aménagements des modes de chasse dans ces espaces permet à la Fédération départementale des chasseurs de la Gironde d'augmenter l'accessibilité du monde de la chasse à une nouvelle population de chasseurs, plus urbaine, dans une perspective de pérennisation de la pratique cynégétique, tandis que la gestion raisonnée des populations de sanglier dans ces milieux pourrait influencer les représentations collectives de la chasse et sa place dans les projets de gestion de territoires.

\section{Les atouts potentiels de la filière}

Au sein de cette nouvelle unité de gestion "périurbaine ", le sanglier change de statut : il passe de nuisible dont la carcasse était détruite à gibier dont la venaison est consommée. En s'inscrivant dans le champ des systèmes agro-alimentaires alternatifs, la valorisation de la venaison prélevée par l'association de chasse périurbaine de Bordeaux par l'intermédiaire d'une filière locale bordelaise se présente comme une opportunité de créer un lien entre le monde urbain et le monde rural, représenté par les chasseurs. Le partage d'une expérience sensorielle avec un public non initié à la chasse à travers la dégusta-

32 Henri Sabarot, président de la Fédération départementale des chasseurs de la Gironde, Assemblée générale de la Fédération départementale des chasseurs de la Gironde du 27 avril 2019.

33 Jérôme Martin, 27 avril 2019, loc. cit.

34 Henri Sabarot, 17 novembre 2019, loc. cit. 
tion de la venaison prélevée localement apparaît comme un outil efficient de communication pour la société de chasse péri-urbaine de Bordeaux, soucieuse de renvoyer une bonne image de la chasse et de recruter de nouveaux chasseurs. Par ailleurs, la mise en place d'une filière de commercialisation de la venaison, impliquant la gestion des déchets de venaison, la collecte organisée des carcasses et leur contrôle systématique par les services vétérinaires compétents au sein d'un centre de traitement, renforcerait le projet de maîtrise du risque sanitaire initié par la Fédération des chasseurs de la Gironde. Enfin en France, les chasseurs alimentent un fonds spécifique géré par les fédérations départementales de chasse servant à financer les mesures de prévention et l'indemnisation des dégâts agricoles causés par le grand gibier. En Gironde, le budget dégât grand gibier sur la saison 2018-2019, dont 80 \% est inféodé au sanglier, atteint un total de 600000 euros. Pourtant, la Fédération parle "d'un bon résultat comparé à d'autres fédérations dont le budget avoisine les 2 millions d'euros. ${ }^{35}$ Aujourd'hui, la situation financière, "plus que saine et qui fait des envieux $»^{36}$ permet la stagnation des prix des cotisations des adhérents pour la saison cynégétique à venir ${ }^{37}$. Néanmoins, les effectifs de chasseurs diminuant chaque année, il est fort possible qu'en l'absence de diminution des indemnisations des dommages aux cultures causés par le grand gibier, l'effort financier par chasseur girondin soit amené à augmenter dans les années à venir. $\mathrm{Ne}$ disposant ni de données sur le poids moyen des sangliers prélevés sur la zone ni d'éléments de négociations avec les différentes parties prenantes d'une filière de commercialisation de la venaison, il est difficile, à ce stade de l'analyse, d'estimer avec précision les retombées économiques potentielles d'un tel projet. Pourtant, l'aspect économique d'une filière venaison mériterait d'être étudié. En effet, par la possible répercussion de ses retombées économiques sur les cotisations des chasseurs, sa mise en place serait cohérente avec l'esprit de chasse populaire et accessible à tous, cher à la Fédération des chasseurs de la Gironde : " chasser en Gironde reste encore une activité financièrement accessible à tous, et nous voulons que cela continue. $»^{38}$

La création d'une filière locale bordelaise contrôlée de commercialisation de la venaison se présente donc comme un moyen susceptible d'appuyer les projets déjà initiés par la Fédération des chasseurs de la Gironde et de répondre ainsi aux objectifs prioritaires annoncés lors de son assemblée générale du 27 avril 2019 (Figure 4).

35 Victor Alcatraz, administrateur responsable de la Commission Grand Gibier, Assemblée générale de la Fédération départementale des chasseurs de la Gironde du 27 avril 2019.

36 Henri Sabarot, président de la Fédération départementale des chasseurs de la Gironde, rencontre préliminaire à Assemblée générale de la Fédération départementale des chasseurs de la Gironde du 4 avril 2020 avec les responsables d'associations et sociétés de chasse de l'entre deux mers, 11 mars 2020.

37 Id.

38 Henri Sabarot, 17 novembre 2019, loc. cit. 


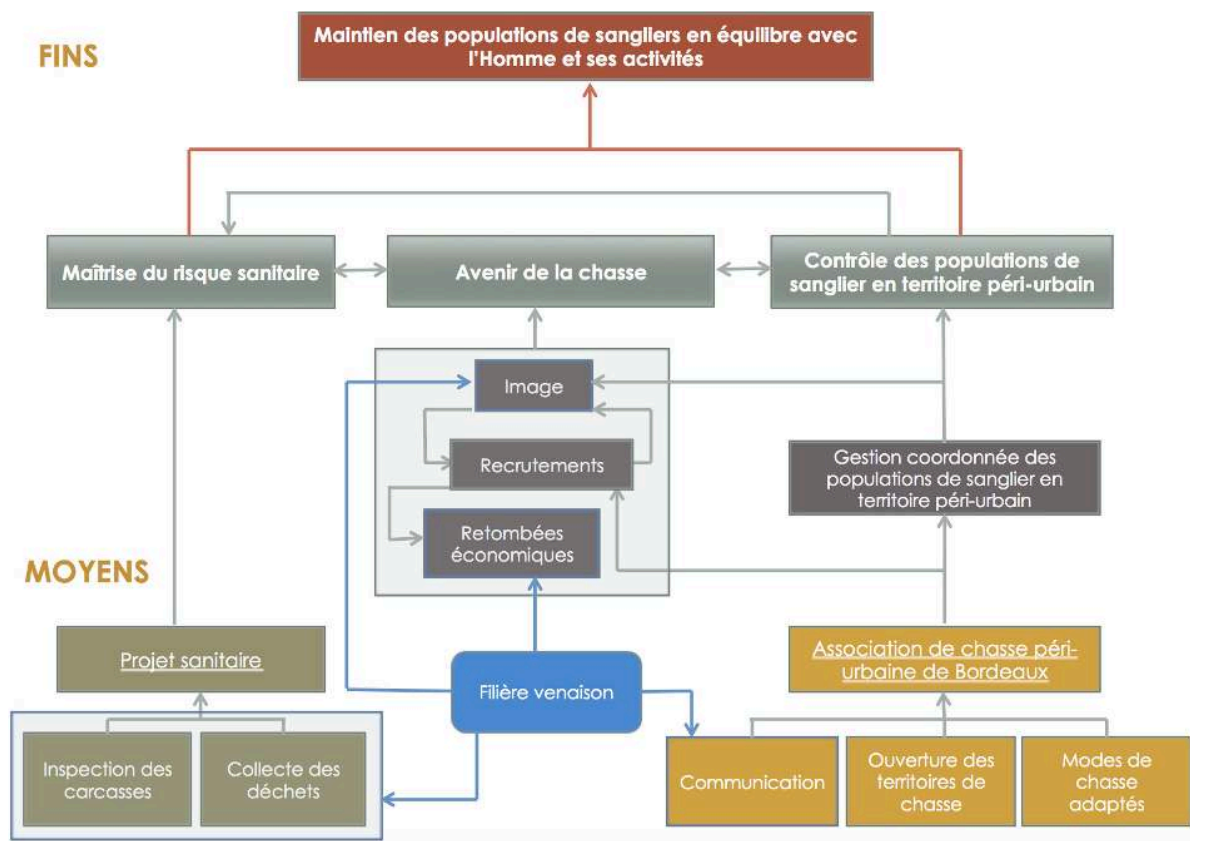

Figure 4: Intégration de la «filière venaison " à l'arbre des objectifs reprenant l’objectif global (encadré rouge), les objectifs spécifiques (encadrés gris) et les projets en cours (encadrés marrons et oranges) de la Fédération départementale des chasseurs de la Gironde (réalisé à partir des discours relevés à l'assemblée générale de la Fédération départementale des chasseurs de la Gironde du 27 avril 2019)

\section{La filière venaison : une opportunité, pour qui ?}

\section{Le point de vue du monde cynégétique}

La réflexion théorique autour du développement d'une filière commerciale contrôlée de la viande de grand gibier prélevé localement révèle les atouts potentiels de celle-ci pour la Fédération départementale des chasseurs de la Gironde. Mais si l'idée a bien été examinée par les gestionnaires cynégétiques, elle a été rapidement abandonnée. Le président de la Fédération départementale des chasseurs, Henri Sabarot, rapporte dans une interview pour le journal Sud-Ouest les difficultés de collecte, le coût et les risques sanitaires qui pourraient découler de la mise en place d'un tel projet ${ }^{39}$. Néanmoins, ces obstacles à la commercialisation du gibier chassé localement n'expliquent pas à eux seuls l'opposition à la création d'une filière venaison. En Gironde, l'accès aux territoires de chasse est gratuit, les chasseurs ne payant pas de redevance aux propriétaires terriens : « La

39 Valérie Deymes. Chasse : pas de commerce de la venaison en Gironde, dit la fédération. Journal Sud-Ouest [En ligne]. Consulté le 30 avril 2020. Disponible sur : https://www. sudouest.fr/2019/05/28/chasse-pas-de-commerce-de-la-venaison-en-gironde-dit-la-federation-6139132-4739.php, 28 mai 2019. 
Gironde est encore un ilot de chasse populaire. L'infirmière chasse avec le chirurgien, le PDG avec l'ouvrier, le grand-père avec le petit-fils. Pourquoi chasset-on pour pas cher? Parce que les agriculteurs, pour la plupart, nous laissent chasser. ${ }^{40}$ L'attribution d'une valeur marchande à la viande de gibier risquerait alors d'entraîner une reconsidération des modalités d'accès aux territoires de chasse : "Chez nous, l'accès [aux territoires] est gratuit. Si on met en place une filière commerciale, on tirerait profit d'une viande d'une propriété pour laquelle on ne débourse rien! Le propriétaire serait tenté de garder pour lui son droit de chasser et de monnayer son gibier directement. L'esprit populaire de la chasse girondine se perdrait alors. ${ }^{41}$ Pour les responsables fédéraux, l'instauration d'un commerce autour de l'activité de chasse pourrait donc constituer une menace à la chasse populaire mais serait également susceptible de favoriser de mauvaises pratiques de gestion des espèces gibiers : «Nous ne voulons pas entendre parler de commerce lié à la chasse car les territoires vont se fermer [...] Certains chasseurs ne pourront plus chasser parce que cela deviendra trop cher pour eux. Et celui qui pourra payer sera prêt à payer des fortunes pour avoir tout le temps un sanglier au bout du fusil. Du coup les sangliers continueront à faire des dégâts, et il y aura toujours des impacts aux activités anthropiques. ${ }^{42}$ Les résultats de l'enquête adressée aux chasseurs girondins montrent que $62,1 \%$ des enquêtés se positionnent eux aussi défavorablement face à la commercialisation de la viande de grand gibier prélevée localement. Ils redoutent en particulier que la valorisation marchande de la venaison favorise le braconnage d'une part (50,7 \% des enquêtés) et soit la source de problèmes entre les chasseurs, les propriétaires terriens et les intermédiaires d'autre part ( $43,4 \%$ des enquêtés). Néanmoins, plus d'un tiers des enquêtés (35\%) estime qu'une telle filière valoriserait la viande de gibier et l'action du chasseur tandis que 31,9\% des répondants considèrent qu'elle permettrait à un large public de déguster du gibier de notre région.

En amont de la commercialisation de la venaison : la chasse en milieu urbain

La réflexion théorique autour de la création d'une filière locale de commercialisation de la viande de gibier prélevé par l'association de chasse périurbaine de Bordeaux interroge la place de la pratique cynégétique dans les territoires urbanisés. La régulation des populations de sangliers dans ces espaces particuliers amène à la rencontre du monde rural représenté par la pratique cynégétique et du monde urbain, désormais confronté aux enjeux liés à la présence de cet animal sauvage. Mais le rapport actuel de l'urbain à l'animal et à la chasse est-il compatible avec un contrôle cynégétique de l'espèce dans ces milieux ?

40 Henri Sabarot, 11 mars 2020, loc. cit.

41 Valérie Deymes, Journal Sud-Ouest, 28 mais 2019, loc. cit.

42 Emmanuel Robin, directeur des actions territoriales de la Fédération départementale des chasseurs de la Gironde, entretien téléphonique du 16 avril 2020. 
Une étude réalisée en 2008 auprès d'habitants berlinois montre que seulement $23 \%$ des résidents s'opposaient à la présence de sangliers dans leur ville. Bien que $44 \%$ des personnes interrogées étaient d'avis qu'il fallait réduire le nombre de sangliers dans la ville de Berlin, $67 \%$ d'entre elles étaient opposées à des méthodes létales de régulation des populations de l'animal ${ }^{43}$. En 2012, la chasse à l'arc avait été introduite à Barcelone. Cette expérience fut rapidement arrêtée en raison de l'opposition de l'opinion publique et finalement, la tranquillisation ou l'utilisation de cages - pièges suivis de l'euthanasie des animaux par des vétérinaires de l'université autonome de Barcelone fut la solution choisie pour la régulation des populations de sangliers dans ces zones ${ }^{44}$. Plus récemment, une enquête menée auprès d'habitants de La Floresta, municipalité de la métropole barcelonaise, fournit les résultats suivants : la grande majorité (93\%) des enquêtés disent apprécier apercevoir des sangliers dans leur environnement, ressentant du respect (61\% des personnes interrogées) et de la sympathie (31\% des enquêtés) pour l'animal. Seulement $37 \%$ des habitants interrogés considéraient que des mesures devaient être prises pour contrôler la présence de sangliers dans ces espaces urbanisés, essentiellement par des opérations de relocalisation des animaux en forêt ${ }^{45}$. À Barcelone et à Berlin, les méthodes létales de régulation des populations de l'animal sont loin de faire l'unanimité auprès des citadins. À Bordeaux, la pratique cynégétique dans les espaces péri-urbains pourrait également se heurter à l'opposition de nombreux résidents : "La chasse n'a jamais été autant attaquée. Depuis six ans, nous mettons des affiches sur les bus de la métropole pour les permis à 0 euro. C'est la première année que nous recevons des attaques sur les réseaux sociaux. J'ai même reçu personnellement une lettre anonyme de menace de mort. On voit bien que l'on a changé de modèle. $»^{46} \mathrm{En}$ effet, la campagne de publicité annuelle déployée début 2020 dans les transports en commun bordelais autour de la prise en charge financière de la formation et de l'examen des candidats au permis de chasser par la Fédération des chasseurs de Gironde a suscité de vives réactions sur les réseaux sociaux : insultes et menaces dirigés vers l'organisation cynégétique ${ }^{47}$. Pour Guillaume Desenfant, directeur de la communication de la Fédération des chasseurs de Gironde, ces réactions s'inscrivent dans une dynamique de chasse bashing, particulièrement

43 York Kotulski, Andreas König, "Conflicts, crises and challenges: wild boar in the Berlin City -a social, empirical and statistical survey ", Natura Croatica, 17(4), 2008, p. 233-246.

44 Séan Cahill, Francesc Llimona, Lluis Cabaneros, Francesc Calomardo, "Characteristics of wild boar (Sus scrofa) habituation to urban aeras in the Collserola Natural Park (Barcelona) and comparison with other locations ", Animal Biodiversity and Conservation, 35(2), 2012, p. 221-233.

45 Carles Conejero et al., Mammalian biology, 2019, loc. cit.

46 Henri Sabarot, 11 mars 2020, loc. cit.

47 LePoint.fr. Gironde : le permis de chasse à 0 euro fait polémique. Journal Le Point, [En ligne]. Consulté le 30 avril 2020. Disponible sur : https://www.lepoint.fr/societe/gironde-le-permisde-chasse-a-0-euro-fait-polemique-29-01-2020-2360409_23.php\#, 29 janvier 2020. 
important en ce moment ${ }^{48}$. Le sentiment est partagé par les chasseurs girondins : $79,7 \%$ des chasseurs enquêtés pensent que la société a, de façon générale, une plutôt mauvaise image de la chasse.

L'inscription de chasseurs dans le paysage urbain bordelais laisse présager l'apparition de tensions entre des acteurs aux pratiques et représentations différentes. Or les chasseurs, bien que chargés de la régulation des populations d'espèces gibier et de l'indemnisation des dégâts agricoles causés par le grand gibier, sont des bénévoles pour qui la pratique de la chasse représente avant tout un loisir. D'après Roland Grange, chasseur de grand gibier en montagne, «Le plaisir de la chasse est lié au territoire dans lequel on chasse. $~^{49}$ L'enquête réalisée auprès des chasseurs girondins montre que seulement $21,9 \%$ des enquêtés disent n'être attachés à aucun territoire en particulier et aimer chasser dans différents territoires. Aussi, 46,6 \% des répondants disent apprécier particulièrement passer du temps sur leur territoire de chasse, qu'ils affectionnent. Si le territoire de chasse revêt une importance particulière pour le chasseur dans la pratique de son loisir, alors la chasse dans le péri-urbain correspond-elle à une chasse "plaisir » ou à une chasse " gestion " ? 62,2\% des chasseurs enquêtés répondent négativement à la question "Auriez-vous envie de chasser dans des territoires urbains et péri-urbains comme autour de la ville de Bordeaux ? " 36,8 \% des répondants préferrent éviter ces nouveaux territoires de chasse en raison des possibles conflits avec les habitants de ces zones et les promeneurs. Un enquêté précise : "Chasser en ville au milieu des maisons !! Zéro plaisir, $100 \%$ danger. Sans compter les insultes... ».

Pour les responsables de la Fédération des chasseurs de la Gironde, il est nécessaire de contrôler les populations de sangliers et les impacts liés à leur présence dans les milieux urbains et périurbains. Mais l'évaluation de la demande de régulation par la société représente un prérequis déterminant la réussite de la mise en place de mesures de gestion de la faune par les politiques publiques et les gestionnaires ${ }^{50}$, en particulier dans des territoires fortement anthropisés. Le recours à différents modes de régulation et en particulier à des méthodes non létales dans cet espace urbanisé mériterait donc d'être évalué. L'étude du contrôle de la fertilité par la castration chimique d'individus reproducteurs pour agir sur la croissance des populations de sangliers fournit notamment des résultats prometteurs ${ }^{51}$. Mais au-delà de leur efficacité

48 Id.

49 Roland Grange, chasseur de grand gibier de montagne, membre du Conseil d'Administration de l'Association départementale des chasseurs de grand gibier de Gironde, entretien du 12 octobre 2018, Pessac.

50 Carles Conejero et al., Mammalian Biology, 2019, loc. cit. ; York Kotulski et Andreas König, Natura Croatica, 2008, loc. cit.

51 Giovanna Massei, Dave Cowan, "Fertility control to mitigate human -wildlife conflicts: a review ", Wildlife Research, 41, 2014, p. 1-21 ; Claudio Oliviero, Lena Lindh, Olli Peltoniemi, 
biomédicale, d'autres facteurs de nature économique, réglementaire et sociale détermineront le développement et l'utilisation de produits contraceptifs sur la faune sauvage ${ }^{52}$. Ici encore, la question de l'acceptation sociale de cette méthode alternative à la chasse se pose : le chasseur se trouve dans un rapport de prédation avec l'animal, la castration chimique perturbe les lois naturelles.

En 1970, Henri Lefebvre annonce l'avènement de la "société urbaine ", marquant la fin de la traditionnelle opposition entre la ville et la campagne ${ }^{53}$. Il rejoint alors le constat d'un autre pionnier, Melvin Weber, qui écrit dans le milieu des années 60 : "l'urbanité cesse d'être la caractéristique exclusive du citadin. Les populations de la suburbie et de l'exurbie figurent parmi les plus urbains des hommes et, de plus en plus, les agriculteurs eux-mêmes participent à la vie urbaine de notre monde. " ${ }^{54}$ De nombreux auteurs ont depuis envisagé le brouillage des formes urbaines et rurales sous le prisme de l'" urbain généralisé ": c'est le cas notamment de Françoise Choay, François Ascher, Thierry Paquot et Michel Lussault ${ }^{55}$. L'esprit urbain s'impose partout, standardisant les activités individuelles et les pratiques culturelles. Mais tandis que certains chercheurs considèrent l'annexion et «l'urbanisation des campagnes " ${ }^{56}$, d'autres géographes soulignent au contraire les spécificités de l'espace rural représentées par les faibles densités de populations humaines, par l'alternance de bâti " diffus » et d'espaces ouverts végétalisés, par la dominance de l'environnement agro-sylvo-pastoral et par les représentations qu'il suscite $^{57}$. L'évolution de ces dernières reflèterait, d'après des auteurs tels que Bernard Kayser ou Nicole Mathieu, l'émergence d'imaginaires revalorisant ces espaces ${ }^{58}$, tandis que pour Emmanuelle Bonerandi et Olivier Deslondes « le

"Immunocontraception as a possible tool to reduce feral pig populations ", Journal of Animal Science, 97, 2019, p. 2283-2290 ; Kim M. Pepin, Amy J. Davis, Fred L. Cunningham, Kurt C. VerCauteren, Doug C. Eckery, "Potential effects of incorporating fertility control into typical culling regimes in wild pig populations ", PLoS ONE, 12(8), 2017, p. 1-23.

52 Kathleen A. Fagerstone, Lowell A. Miller, Gary Killian, Christi A. Yoder, « Review of issues concerning the use of reproductive inhibitors, with particular emphasis on resolving humanwildlife conflicts in North America ", Integrative Zoology, 5(1), 2010, p. 15-30.

53 Henri Lefebvre, La Révolution urbaine, Paris, Gallimard, 1970, 256 p.

54 Melvin M. Webber, L'Urbain sans lieu ni bornes, trad. Xavier Guillot, La Tour-d'Aigues, Éditions de l'Aube, 1996, $123 \mathrm{p}$.

55 Hervé Marchal, Jean-Marc Stébé, "Vers l'urbanisation généralisée des villes », in Hervé Marchal, Jean-Marc Stébé (éds), Les grandes questions sur la ville et l'urbain, Paris, Presses Universitaires de France, 2014, p. 45-49.

56 Étienne Juillard, «L’urbanisation des campagnes », Études rurales, 49-50, 1961, p. 5-9.

57 Laurent Rieutort, "La géographie française et la question rurale ", in Martine Guibert, Yves Jean (éds), Dynamiques des espaces ruraux dans le monde, Paris, Armand Colin, 2011, p. $17-45$. 
rural comme idée, concept ou objet de désir, ne s'est jamais mieux porté " $^{59}$. La ruralité n'est pas une réalité géographique circonscrite dans l'espace rural, mais bien une construction sociale reposant sur des représentations, modes de vie et pratiques en constante évolution et sous influence urbaine. Néanmoins, le mouvement ne semble pas unilatéral : la valorisation des espaces ouverts dans les projets récents de planification urbaine $e^{60}$, l'intégration de l'agriculture aux politiques et projets territoriaux urbains ${ }^{61}$ ou encore le retour d'animaux d'élevage au cœur des villes ${ }^{62}$ reflètent le retour, au cours des dernières années, de pratiques et paysages relevant de la ruralité dans le monde urbain. Les résultats préliminaires de la recherche en cours tendent à montrer que le sanglier, espèce forestière, s'adapte à l'environnement urbain de la métropole bordelaise. Profitant de la quiétude des espaces ouverts non chassés traversant la ville, dans lesquels « il ne s'agit pas seulement de protéger les espaces ayant une valeur environnementale reconnue, mais de favoriser le maintien des continuités, des corridors de circulation des espèces et des flux naturels ${ }^{63}$, le sanglier s'urbanise. Nuisible soumis à destruction administrative, le sanglier " urbain " devient, en 2019 espèce gibier dont la chasse est coordonnée par une nouvelle association cynégétique, urbaine. Le monde cynégétique s'adapte, les pratiques s'urbanisent dans ces espaces. Si « l'intégration d'espaces de faible densité dans les systèmes urbains ne peut suffire à qualifier le mouvement de ruralisation des villes ${ }^{64}$, qu'en est-il de la présence du sanglier et de la chasse en ville ?

À Bordeaux, le sanglier et la chasse font désormais partie de l'environnement urbain. Dans un contexte de revendications sociétales d'une alimentation saine, traçable et locale ${ }^{65} \mathrm{~d}^{\prime}$ une part et d'augmentation constante des prélèvements de sangliers d'autre part, la réflexion autour de l'intégration d'une filière locale de commercialisation de la " venaison bordelaise " au projet de régulation cynégétique des populations urbaines de sangliers nous est apparue pertinente. Le phénomène d'urbanisation du sanglier ainsi que les moyens choisis pour la gestion de ses populations amènent à la confrontation

59 Emmanuelle Bonerandi, Olivier Deslondes, "Où va la géographie rurale ", Géocarrefour, 83(4), 2008, p. 255-258.

60 Mayté Banzo, "L'espace ouvert pour recomposer avec la matérialité de l'espace urbain ", Articulo - Journal of Urban Research, 6, 2015, p. 1-16.

61 Mayté Banzo, Laurent Couderchet, "Intégration de l'agriculture aux politiques et projets territoriaux urbains. Le cas bordelais ", Sud-Ouest Européen, 35, 2013, p. 5-16.

62 Claire Delfosse, Adrien Baysse-Lainé, « Lélevage en milieu urbain entre nature et nourriture. Le cas des métropoles de Lyon et Grenoble ", Géocarrefour, 92(3), 2018, p. 1-20.

63 Mayté Banzo, Dominique Prost, « Aménagements paysagers et renouvellement urbain dans la périphérie bordelaise ", M@ppemonde, 93, 2009, p. 1-19.

64 Mayté Banzo, 2015, loc. cit.

65 Monique Poulot, «Du vert dans le périurbain. Les espaces ouverts, une hybridation de l'espace public (exemples franciliens) ", Espaces Temps.net, 2013. Disponible sur : https://hal.archivesouvertes.fr/hal-00974374. 
du monde urbain et du monde rural, représenté à la fois par l'animal et par les chasseurs. La consommation de " gibier urbain » se révèle alors susceptible de réunir ces deux mondes autour d'une expérience sensorielle. Aussi, par la valorisation et le partage de sa viande, la mort de l'animal devient certes visible, mais prend un sens différent de celui de l'éradication d'un nuisible par destruction administrative.

Si la première partie de l'article adopte une démarche purement pragmatique dégageant les moyens nécessaires à la mise en place d'une filière facilitant le traitement et la mise sur le marché de la venaison prélevée en Gironde, certains atouts potentiels de la filière avancés dans la suite de l'article relèvent une dimension éthique. En s'inscrivant dans une logique de justification morale de la mise en place d'une filière commerciale de la viande de gibier prélevé à des fins de régulation, la réflexion proposée ici dépasse le registre du fait et entre dans celui des valeurs. Mais le monde cynégétique et les habitants non-chasseurs de l'espace urbain considéré partagent-ils le système de valeurs sous-jacent à notre réflexion, largement influencé par notre système de représentations que notre expérience de vétérinaire a contribué à construire ? L'association d'une filière venaison à la régulation cynégétique des populations urbaines de sangliers est-elle la «bonne norme » à adopter pour tous? Répondre à cette dernière question par l'affirmative reviendrait à considérer l'existence d'une morale universelle. Pourtant, dans la situation particulière analysée ici, les arguments " rationnels " avancés en faveur de la création de la filière ne suffisent pas à contrer les oppositions au projet, elles aussi d'ordre moral. Elles s'appuient, pour les acteurs cynégétiques, sur une éthique conséquentialiste : la commercialisation de la venaison menacerait la chasse populaire d'une part, et pourrait favoriser l'essor de mauvaises pratiques de gestion des populations d'espèces gibier d'autre part. Pour la société urbaine, les hypothèses d'opposition au projet portent sur une autre forme d'éthique : celle de la conviction. Ici, ce sont les pratiques cynégétiques de régulation de l'animal en amont de la consommation de sa viande qui posent question. D'autres scénarios de gestion de l'espèce sont envisageables. Leur accueil par la société serait alors tout autant sujet à discussion. C'est le cas de la castration chimique, qui, de la même façon que les mesures d'abattages administratives, invisibiliserait les moyens de contrôle de la croissance des populations de l'animal, mais dont l'introduction dans les pratiques de gestion de la faune sauvage représenterait par ailleurs une manifestation de la victoire de la science et de la technique sur la nature. Par ailleurs, à une époque où la nature est de plus en plus valorisée, le " vivre-ensemble " est-il un scénario envisageable ?

"La diversité morale se niche au cœur même de notre société et des nombreuses sensibilités et appartenances qui la composent. " ${ }^{66}$ Dans notre exemple, le pluralisme moral semble être influencé par la divergence dans les pratiques et représentations relatives à la nature, à la chasse et à l'animal. Le système de valeur n'est pas pur produit de la raison, il varie suivant les

66 Catherine Halpern, "La pensée morale à l'épreuve de la diversité ", in Nicolas Journet (éd.), La Morale, Auxerre, Sciences Humaines Éditions, 2012, p. 127 à 132. 
systèmes de représentations des acteurs. Mais alors, quelle est la «bonne morale " ? Jürgen Habermas dépasse l'opposition entre l'universalisme et le relativisme moral, en développant le concept de l'éthique de la discussion. Selon lui, il s'agit de définir la norme juste, celle obtenue dans le respect des règles de la discussion entre les personnes concernées ${ }^{67}$. Dans cette perspective, la rencontre des différents acteurs pour la définition d'un projet de gestion des populations de "sanglier urbain " pourrait faire émerger de nouvelles normes, et ainsi participer à la construction d'une nouvelle urbanité, entre ville et campagne. Relevons que les théories du modèle délibératif font l'objet de réflexions de la part de certains auteurs. D'abord, la visée d'un consensus pourrait avantager la position de certains acteurs avançant des arguments considérés comme rationnels et ainsi déséquilibrer la discussion. Aussi, parmi les finalités de la délibération, des approches différentes de celle de l'universalité d'un accord sont envisagées, parmi lesquelles celle de l'approfondissement des controverses à travers la confrontation des différents points de vue relatifs à la question délibérée ${ }^{68}$. Cette conception de la démocratie participative ouvre alors des perspectives de réflexions sur le rôle du conflit dans le changement social. Finalement, la réflexion autour de la création d'une filière de commercialisation de la venaison en Gironde s'inscrit dans une problématique plus large interrogeant l'histoire de l'humain avec le sanglier dans le contexte urbain : le sanglier sera-t-il accepté dans le paysage urbain dans le futur ? La gestion cynégétique des populations de l'espèce aura-t-elle sa place dans les projets de territoires urbains et péri-urbains?

Carole Marin

Unité Mixte de Recherche Passages - CNRS 5319 École Doctorale de Bordeaux Montaigne-Humanités carole.marin@u-bordeaux-montaigne.fr

La réflexion et les résultats exposés dans cet article s'inscrivent dans le cadre d'une thèse de doctorat en géographie en cours réalisée par Carole Marin. Vétérinaire praticienne de 2010 à 2018, elle débute sa thèse au laboratoire Passages UMR 5319 du CNRS et de l'Université de Bordeaux Montaigne en septembre 2018, intitulée "Sauvage en ville. Le sanglier bordelais ". L'étude porte sur l'analyse du phénomène de pénétration de populations de sangliers au sein de l'enveloppe urbaine bordelaise, considérée comme un espace de cristallisation des enjeux socio-spatiaux liés à la plasticité de l'espèce. L'approche est globale et interdisciplinaire : la thèse, inscrite en géographie, convoque également les sciences écologiques et vétérinaires. Le travail de recherche, financé par un contrat doctoral ministériel, s'effectue sous la direction de Laurent Couderchet, Professeur des universités en géographie, et de Nicolas Lemoigne, Maitre

67 Jürgen Habermas, De l'éthique de la discussion, Paris, Les éditions du cerf, 1992, 208 p.

68 Loïc Blondiaux, "Démocratie délibérative vs démocratie agonistique ? Le statut du conflit dans les théories et les pratiques de participation contemporaines ", Raisons politiques, 30, 2008, p. 131-148. 
de conférences, de l'Université Bordeaux Montaigne et rattachés à l'unité mixte de recherche Passages. Enfin, le travail reçoit le soutien matériel, technique et humain de la Fédération départementale des chasseurs de la Gironde.

\title{
Résumé
}

En Gironde, il n'existe pas de filière commerciale de la viande de gibier prélevé sur le département. Pourtant, l'augmentation continue des tableaux de chasse au sanglier couplée à la diminution des chasseurs girondins laisse présager l'atteinte des limites du monde de la chasse à préparer et à consommer l'intégralité de la viande de grand gibier qu'il prélève. Dans cet article, nous exposons les moyens nécessaires à la création d'une filière locale de traitement et de commercialisation de la venaison de sanglier prélevé par une nouvelle organisation cynégétique, urbaine : l'association de chasse périurbaine de Bordeaux. Nous discutons des avantages d'un tel projet mais également des éventuels obstacles à sa mise en place. Finalement, nous soulevons la question de la place de la pratique cynégétique dans des territoires urbanisés.

\section{Mots-clés}

Sanglier, chasse, filière venaison, territoires cynégétiques, interface urbain-rural, faune sauvage urbaine, gestion de la faune sauvage.

\begin{abstract}
In Gironde, there is no local venison sector. But the continuous increase in wild boar hunting bags associated with the decline in hunters population suggests that hunting world could reach its limits to prepare and consume all the big game meat it takes. This article proposes to expose the means for the creation of a local treatment and marketing sector of the wild boar venison harvested by a new urban hunting organization: Bordeaux peri-urban hunting association. We discuss the advantages of such a project and the possible obstacles to its implementation. Finally, we interrogate the place of hunting in urbanized areas.
\end{abstract}

\section{Keywords}

Wild boar, hunting, venison sector, hunting territories, urban-rural interface, urban wildlife, wildlife management. 